\title{
Investigation into the reasons for preventable drug related admissions to a medical admissions unit: observational study
}

\author{
R L Howard, A J Avery, P D Howard, M Partridge
}

Qual Saf Health Care 2003;12:280-285

See end of article for authors' affiliations

Correspondence to: Mrs R L Howard, Division of Primary Care, School of Community Health Sciences, University Hospital, Nottingham NG7 2UH, UK:

Rachel.howard@

broxtowehucknall-pct.nhs.uk

Accepted for publication 29 May 2003
$\mathrm{R}$ ecent reports in the USA ${ }^{1}$ and the $\mathrm{UK}^{2}$ highlight the problem of drug related morbidity and the need to find ways to prevent medical errors. These reports suggest that preventable drug related admissions require particular attention and, in 2002, two systematic reviews were published on this topic. ${ }^{3}$ Winterstein et al ${ }^{3}$ identified 15 studies of preventable drug related hospital admissions and found a median of $7.1 \%$ of admissions to be drug related, and $59 \%$ of these to be preventable. Beijer $e t a l^{4}$ reviewed 68 studies, 12 of which looked at preventable admissions. This review highlighted some of the limitations of previous research and emphasised the need for further studies looking at the preventability of drug related admissions and documentation of the drugs responsible for these admissions. This sort of information is needed if we are to develop effective strategies to prevent drug related admissions. In addition, it is important to identify some of the underlying causes of preventable admissions.

We have addressed some of these issues by conducting a large study of drug related morbidity detected by pharmacists on a medical admissions unit in the UK. We have defined drug related morbidity as adverse drug reactions, ${ }^{5}$ failure to optimise treatment, unintentional overdose, and adherence problems. Admissions associated with intentional overdoses and drugs of abuse were excluded.

The objectives of our study were to:

- estimate the proportion of admissions to an acute medical admissions unit that were associated with drug related morbidity and the proportion of these admissions that were potentially preventable;

- identify the drugs associated with the preventable drug related admissions

- classify preventable drug related admissions by the underlying causes-for example, prescribing problems, monitoring problems, adherence problems and supply problems.

\section{METHODS}

Selection of participants

The local research ethics committee approved the study. All patients admitted to the medical admissions unit (box 1) and seen by a pharmacist between 1 January and 30 June 2001 were included in the study. The pharmacists saw patients as part of their routine work Mondays to Fridays. They recorded details of all patients seen and reported patients with any suspected drug related morbidity to the principal investigator

Box 1 Description of the Medical Admissions Unit at Queeens Medical Centre, Nottingham

The medical admissions unit at Queens Medical Centre (QMC), Nottingham is a 30 bed unit which receives acute medical admissions from general practitioners and the A\&E department. A small number of admissions come from other hospitals or wards within the QMC (these were not included in the study). The ward is covered Monday to Friday by three admissions ward pharmacists (all have a clinical diploma) between 07.00 and 19.00 hours. The pharmacists are responsible for checking the medication histories of patients admitted (by reviewing patients' own medication, questioning patients, contacting general practice surgeries and nursing homes, etc), as well as supplying medication and ensuring the safety of the prescribing on the unit. Part of this role inevitably involves identifying drug related morbidity.

There are two consultant-led multidisciplinary ward rounds each day (morning and evening) which are attended by one of the admissions unit pharmacists.

Overnight and at weekends the admissions unit is covered by a limited pharmacy service. 
8781 admissions between 1 January 2001 and 30 June 2001

$\longrightarrow 4688$ admissions not screened by the admissions ward pharmacists

3784 admissions screened by the admissions ward pharmacists and 309 by the principal investigator (total 4093 admissions screened)

3512 admissions screened as not drug related by the admissions ward pharmacists and principal investigator

581 admissions screened as possibly drug related by the admissions ward pharmacists and principal investigator

$\longrightarrow 170$ possible drug related admissions excluded after follow up by the principal investigator

411 admissions assessed by the panel

$\longrightarrow 146$ admissions classified as not drug related

1

265 admissions classified as drug related

87 admissions classified as drug related and not

$\longrightarrow$ preventable

178 admission classified as drug related and preventable

Figure 1 Flow of patients through the study.

\section{Box 2 Details included in the case summaries}

- Unique identifier

- Patient's age

- Sex

- Date of admission

- Date of discharge

- Presenting complaint

- Medication history (before admission to hospital)

- Medication on discharge

- Events leading up to the admission

- Summary of hospital treatment

- Test results

- Diagnosis made by the physicians caring for the patient in hospital

(RH). Similar methods have been used successfully in at least five previous studies. ${ }^{6-10}$

The principal investigator assessed possible cases using a combination of:

- medical note review (throughout the admission and on discharge);

- contact with general practitioners, where necessary, to obtain medication histories, test results, and information regarding the management of patients in primary care;

- interviewing patients, where possible, about the type and duration of symptoms and medication use, using an interview schedule. Those not interviewed included patients discharged home before being seen by the principal investigator, patients too unwell to be interviewed, and patients unable to speak English.

Following assessment by the principal investigator, some cases were excluded from further review (fig 1). These included cases where further investigation suggested that drug related morbidity was unlikely-for example, a low probability VQ scan in a case of suspected pulmonary embolism in a woman taking a combined oral contraceptive. In order to ensure that some patients were included in the study who had been admitted at weekends, the principal investigator identified patients with potential drug related morbidity through medical note review and patient interview on alternate weekends (fig 1).

\section{Classification of potential drug related admissions}

Following discharge from hospital, the principal investigator prepared detailed case summaries on patients with suspected
Box 3 Criteria used to review possible drug related admissions cases

Amended Hallas criteria for causality

(1) Known adverse drug reaction, toxic reaction, response to omission of treatment or inadequate treatment.

(2) Reasonable temporal relationship between commencement or cessation/omission of treatment and onset of problem.

(3) Risk of further problems likely to be reduced by dose reduction or increase, discontinuation, closer monitoring or commencement of treatment.

(4) Not explained by any other known condition of predisposition to the patient, or this condition/predisposition is likely to be exacerbated by the presence/absence of the drug.

(5) For drug toxicity:

- symptoms re-appeared upon re-exposure;

- laboratory tests showed toxic drug levels or drug induced metabolic disturbances that explained the symptom;

- symptoms resolved on dose reduction or discontinuation of the drug.

For drug omission:

- symptoms resolved upon re-introduction of the drug or dose increase.

If $\mathbf{5}$ criteria fulfilled then definite.

If $\mathbf{4}$ criteria fulfilled then probable

If $\mathbf{3}$ criteria fulfilled then possible

If $\mathbf{2}$ or less criteria fulfilled then either, not drug related or unevaluable

Hepler criteria for preventability

(1) Drug related morbidity (DRM) preceded by a recognisable drug therapy problem (DRP).

(2) Given the DRP, the DRM would have been reasonably foreseeable.

(3) The cause of DRM would have been identifiable with reasonable probability (Hallas criteria probable or definite for causality).

(4) The cause of the DRM could have been reasonably controllable within the context and objectives of treatment.

All four criteria must be fulfilled to confirm preventability.

Contribution of drug related problem to hospital admission

Score $=3$ (dominant): the suspected symptoms were the main reason for admission and no other symptoms contributed significantly.

Score $=2$ (partly contributing): the suspected symptoms played a substantial role in admission, but other factors also contributed significantly.

Score $=1$ (less important): the suspected symptoms played a minor or uncertain role, and the patient would probably have been admitted without them

Score $=0$ (not contributing): other symptoms/circumstances were the reason for hospitalisation.

\section{Hepler definitions for classification of drug related} admissions

Score $=1$ : inappropriate prescribing.

Score $=2$ : inappropriate delivery (unavailable when needed, inappropriate formulation, failure to administer, dispensing error).

Score = 3: inappropriate behaviour by the patient (non-compliance).

Score $=4$ : patient idiosyncrasy (response to drug, mistake, or accident).

Score $=5$ : inappropriate monitoring .

Score $=6^{*}$ : potentially preventable with interventions which are not standard care at present.

*Category 6 is additional to the original Hepler classification 
Table 1 Primary diagnoses of patients admitted to the medical admissions unit where there was a statistically significant difference between the numbers of patients seen and not seen by a pharmacist.

\begin{tabular}{llll}
\hline $\begin{array}{l}\text { Primary diagnoses classified by ICD-10 } \\
\text { chapter heading }\end{array}$ & $\begin{array}{l}\text { Patients seen by } \\
\text { pharmacist }(n=3799)\end{array}$ & $\begin{array}{l}\text { All patients } \\
\text { admitted }(n=7962)\end{array}$ & p value* \\
\hline Certain infectious and parasitic diseases & $101(2.7)$ & $172(2.2)$ & 0.003 \\
Diseases of the nervous system & $153(4.0)$ & $283(3.6)$ & 0.029 \\
Mental and behavioural disorders & $84(2.2)$ & $151(1.9)$ & 0.049 \\
$\begin{array}{l}\text { Diseases of the respiratory system } \\
\text { Diseases of the skin and subcutaneous tissue }\end{array}$ & $702(18.5)$ & $1305(16.4)$ & $<0.001$ \\
$\begin{array}{l}\text { Diseases of the musculoskeletal system and } \\
\text { connective tissue }\end{array}$ & $106(2.8)$ & $163(2.0)$ & $<0.001$ \\
& & $410(5.1)$ & $<0.001$
\end{tabular}

Values are no $(\%)$.

*Based on $\chi^{2}$ tests (1 degree of freedom).

Table 2 Reviewer agreement in scoring case summaries calculated using kappa and intraclass correlation coefficients

\begin{tabular}{|c|c|c|c|c|}
\hline & $\begin{array}{l}\text { Kappa for } \\
\text { RH*TA }\end{array}$ & $\begin{array}{l}\text { Kappa for } \\
\text { RH*PH }\end{array}$ & $\begin{array}{l}\text { Kappa for } \\
\text { TA*PH }\end{array}$ & $\begin{array}{l}\text { Intraclass correlation } \\
\text { coefficient }(95 \% \mathrm{CI})\end{array}$ \\
\hline Causality & 0.81 & 0.81 & 0.77 & 0.88 (0.86 to 0.90$)$ \\
\hline Preventability & 0.77 & 0.77 & 0.69 & $0.74(0.70$ to 0.78$)$ \\
\hline Contribution to admission & 0.74 & 0.87 & 0.76 & 0.80 (0.78 to 0.83$)$ \\
\hline $\begin{array}{l}\text { Cause of suboptimal } \\
\text { outcome }\end{array}$ & 0.78 & 0.71 & 0.67 & 0.75 (0.71 to 0.78$)$ \\
\hline
\end{tabular}

drug related morbidity (box 2). These summaries provided reviewers with information on temporal relationships between medications and symptoms, the nature of patients' symptoms, medication changes resulting from the admission, and the diagnoses made by physicians caring for the patients. The case summaries were independently scored by a hospital physician $(\mathrm{PH})$, an academic general practitioner with a special interest in prescribing (AA), and a clinical pharmacist (RH). Each reviewer scored the cases using explicit criteria for causality, ${ }_{11}^{11}$ preventability, ${ }_{12}^{12}$ contribution to the admission, ${ }^{11}$ and classification of the underlying cause of the drug related morbidity $^{12}$ (box 3). All the criteria were validated in pilot study work where 46 possible cases of drug related morbidity were reviewed to ensure the criteria were reliable and gave reasonable agreement between the reviewers. After scoring the cases the reviewers met up and, in keeping with standard practice in this field, ${ }^{3}$ a majority decision was used to classify the cases where there was disagreement on any of the review criteria.

Admissions were classified as drug related if two or more reviewers scored 4 or 5 using the amended Hallas criteria and judged the drug to have made a dominant or partial contribution to the admission. Admissions were classified as potentially preventable if the above criteria were fulfilled and two or more reviewers scored 4 using the Hepler criteria for preventability.

\section{Recording of data}

For all admissions to the medical admissions unit age, sex, and date of admission were recorded on an ACCESS 97 database. The hospital routinely records details of primary diagnoses of patients admitted, classified according to the International Classification of Diseases and Related Health Problems, 10th revision. ${ }^{13}$ These data were available for $7962(91 \%)$ of the admissions in our study and were imported from the hospital database into the study database.

To identify the patients seen by a pharmacist, a record was kept on the database. Data obtained from patient interviews, medical record reviews, and GP contacts were also recorded on the database and were used to generate case summaries for the reviewers. The judgements made by individual reviewers and the final classifications for each case were double entered onto the database. In addition, details of the drugs that were thought to have contributed to the admissions were recorded and were grouped by British National Formulary codes. ${ }^{14}$

\section{Analysis of data}

Data were exported to SPSS version 10.0 for statistical analysis. To assess differences between the groups of patients seen and not seen by a pharmacist, patient characteristics were compared using an independent $t$ test for age on admission and $\chi^{2}$ tests for sex, day of admission, and primary diagnosis (available for 7962 (91\%) of all patients admitted). To assess inter-reviewer reliability, case review scores were compared using Cohen's kappa and intraclass correlation coefficients (moderate to substantial agreement is indicated by kappa $0.41-0.80) .{ }^{15}$ The proportion of patients considered to have a drug related admission was calculated as the percentage of patients seen by a pharmacist who were classified as having definite or probable drug related morbidity that had made a dominant or partial contribution to the admission. We also calculated the proportion of drug related admissions that were considered potentially preventable. The proportion of patients considered to have a potentially preventable drug related admission was calculated as the percentage of patients seen by a pharmacist who were judged to have a preventable drug related admission.

\section{RESULTS}

\section{Patient characteristics}

The flow of patients through the study is shown in box 2. Of the 8781 patients admitted to the medical admissions unit, $4093(47 \%)$ were seen by a pharmacist. The mean (SD) age of patients seen was 62.6 (20.7) years and the mean (SD) age of patients not seen by a pharmacist was 62.0 (20.6) years. Of the patients seen by a pharmacist 2002 (49\%) were female and 2091 (51\%) were male, and of the patients not seen by a pharmacist 2399 (51\%) were female and 2289 (49\%) were male.

Comparing patients who were either seen or not seen by a pharmacist, there were no significant differences in age 
Table 3 Drug classes most frequently associated with preventable drug related admission due to prescribing problems

\begin{tabular}{|c|c|c|c|}
\hline British National Formulary class & Adverse drug event & Prescribing problem & $\begin{array}{l}\text { Number } \\
\text { of cases }\end{array}$ \\
\hline \multirow[t]{3}{*}{ Non-steroidal anti-inflammatory drugs } & Gastrointestinal toxicity & $\begin{array}{l}\text { Prescription in patients with two or more risk factors without } \\
\text { gastrointestinal prophylaxis* }\end{array}$ & 21 \\
\hline & Renal tubular necrosis & $\begin{array}{l}\text { Concurrent prescription of two full dose non-steroidal } \\
\text { anti-inflammatory drugs without monitoring renal function }\end{array}$ & 1 \\
\hline & & Subtotal & 22 \\
\hline \multirow[t]{3}{*}{ Antiplatelet drugs } & Gastrointestinal toxicity & $\begin{array}{l}\text { Prescription in patients with two or more risk factors without } \\
\text { gastrointestinal prophylaxis* }\end{array}$ & 13 \\
\hline & Thrombotic event & Failure to prescribe in patients needing secondary prevention & 4 \\
\hline & & Subtotal & 17 \\
\hline \multirow[t]{6}{*}{ Beta-adrenoceptor blocking drugs } & Congestive cardiac failure & $\begin{array}{l}\text { Prescription of standard dose beta-blocker in patient with known } \\
\text { congestive cardiac failure }\end{array}$ & 2 \\
\hline & & Co-prescription of atenolol with verapamil* & 1 \\
\hline & Tachycardia & Sudden cessation & 1 \\
\hline & Bleeding oesophageal varices & Cessation without prescription of alternative & 1 \\
\hline & Chest pain & $\begin{array}{l}\text { Failure to maximise anti-anginal therapy despite ongoing } \\
\text { symptoms over a period of time* }\end{array}$ & 2 \\
\hline & & Subtotal & 7 \\
\hline \multirow[t]{3}{*}{ Antiepileptics } & Fitting & Subtherapeutic prescription & 4 \\
\hline & & Inappropriate cessation & 2 \\
\hline & & Subtotal & 6 \\
\hline Other & & & $38 \dagger$ \\
\hline
\end{tabular}

$(\mathrm{p}=0.168)$, a weakly significant difference in $\operatorname{sex}\left(\chi^{2} 24.7\right.$, df 1 , $\mathrm{p}=0.031$ ), and a marked difference in terms of day of admission $\left(\chi^{2} 568\right.$, df $1, p<0.001 ; 3070(75 \%)$ of the patients seen by a pharmacist were admitted Monday to Thursday). Comparison of primary diagnoses indicated that those with infections, nervous system diseases, mental disorders, and respiratory diseases were over-represented in the study, and those with skin and musculoskeletal problems were under-represented (table 1$).^{13}$

\section{Inter-reviewer reliability}

Kappa and intraclass correlation coefficients showed good to excellent agreement between the reviewers (table 2).

\section{Proportion of admissions considered drug related}

Drug related morbidity was judged to be the cause of 265 $(6.5 \%)$ of the admissions seen by a pharmacist, and 178 (67\%) of these were judged to be potentially preventable. The main underlying causes of preventable drug related admissions were prescribing problems $(n=63(35 \%))$, monitoring problems $(n=46(26 \%))$, and adherence problems $(n=53(30 \%))$. Problems with administration of medication were infrequent $(n=9(5 \%))$. Aspirin associated gastrointestinal bleeds were classified as "potentially preventable with interventions which are not standard care at present" $(\mathrm{n}=7(4 \%))$.

The drugs most commonly associated with preventable drug related admissions can be seen in tables 3, 4, and 5 . These account for $105(60 \%)$ of the preventable drug related admissions (details of all drugs associated with the preventable admissions are available on the QSHC website at www.qshc.com/supplemental).

\section{DISCUSSION}

In this large observational study we found that $6.5 \%$ of admissions screened by pharmacists on a medical admissions unit were judged to be drug related and $67 \%$ of these were considered preventable. The drugs most commonly associated with potentially preventable drug related admissions were NSAIDs, low dose aspirin, beta-blockers, antiepileptics, diuretics, sulphonylureas, digoxin, inhaled corticosteroids, nitrates, and insulin. These admissions were mainly attributed to problems with prescribing, monitoring, and patient adherence.
In order to minimise the risk of misclassifying cases, we used a methodology which compares well with the most rigorous studies previously reported. ${ }^{3816}$ We used explicit criteria to guide the assessment of cases and a three member panel of reviewers to independently classify each case. Despite the varied backgrounds of the reviewers, kappa and intraclass correlation coefficients showed good to excellent levels of agreement. Only those admissions where the drug related morbidity was judged to be the dominant cause, or partially contributing, to the admission were included as drug related admissions. Also, when we classified drug related admissions as preventable, it was our view that the problems identified were predictable and controllable in terms of the treatment (Box 4). Nevertheless, our results could be questioned in terms of the extent to which they reflect the true preventability of the observed outcomes. For example, a recent study of hospital deaths that were attributed to medical error ${ }^{18}$ suggested that in many cases the error made relatively little difference to expected fatal outcomes in seriously ill patients. While these results are not directly comparable to our study, we recognise that some of the patients in our study were at very high risk of hospital admission regardless of any preventable medication related adverse event.

In our study we reviewed only those patients seen by a pharmacist on the admissions unit. This means that our estimates of the proportion of admissions that were drug related and preventable need to be treated with some caution. The patients we studied were not fully representative of all patients admitted. However, the differences between the groups seen and not seen by a pharmacist were relatively small and, had we studied all the admissions, it is unlikely that the results would have been substantially different. In addition, our findings were similar to median figures from a recent systematic review. ${ }^{3}$

In recent years there have been calls for researchers to move beyond simply reporting preventable adverse events to finding ways of improving patient safety through avoidance of these events. ${ }^{12}$ It has been suggested that an approach focusing on systems failures is likely to be most effective. ${ }^{12} 19$ The key to starting this process is to find out which types of problem are most important. ${ }^{20}$

Almost $60 \%$ of the preventable drug related admissions that we found are shown in tables 3-5. These data describe the drugs most commonly associated with admissions. We have recently completed a systematic review of interventions aimed 
Table 4 Drug classes most frequently associated with preventable drug related admissions due to monitoring problems

\begin{tabular}{|c|c|c|c|}
\hline $\begin{array}{l}\text { British National Formulary } \\
\text { class }\end{array}$ & Adverse drug event & Problem & $\begin{array}{l}\text { Number } \\
\text { of cases }\end{array}$ \\
\hline \multirow[t]{5}{*}{ Loop diuretics } & $\begin{array}{l}\text { Overdiuresis causing dehydration } \\
\pm \text { renal failure } \pm \text { electrolyte imbalance }\end{array}$ & Failure to monitor fluid balance, renal function, electrolytes etc* & 7 \\
\hline & Congestive cardiac failure & $\begin{array}{l}\text { Failure to monitor following cessation of angiotensin converting enzyme } \\
\text { inhibitor and diuretics* }\end{array}$ & 1 \\
\hline & Dizziness & Unnecessary polypharmacy* & 1 \\
\hline & Hyponatraemia & Failure to check electrolytes & 1 \\
\hline & & Subtotal & 10 \\
\hline \multirow[t]{5}{*}{ Potassium sparing diuretics } & $\begin{array}{l}\text { Overdiuresis causing dehydration } \\
\pm \text { renal failure } \pm \text { electrolyte imbalance }\end{array}$ & Failure to monitor fluid balance, renal function, electrolytes etc* & 5 \\
\hline & Hyponatraemia & & 1 \\
\hline & Hyperkalaemia & & 1 \\
\hline & Dizziness & Unnecessary polypharmacy* & 1 \\
\hline & & Subtotal & 8 \\
\hline \multirow[t]{3}{*}{ Sulphonylureas } & Hypoglycaemia & Failure to monitor blood sugar and renal function & 5 \\
\hline & Hyperglycaemia & Failure to monitor blood sugar & 3 \\
\hline & & Subtotal & 8 \\
\hline \multirow[t]{4}{*}{ Cardiac glycosides } & Digoxin toxicity & Failure to monitor renal function and/or digoxin levels at least annually. & 4 \\
\hline & Fast atrial fibrillation & Failure to ensure that digoxin levels were therapeutic & 1 \\
\hline & Dizziness & Unnecessary polypharmacy* & 1 \\
\hline & & Subtotal & 6 \\
\hline \multirow[t]{3}{*}{ Thiazide and related diuretics } & $\begin{array}{l}\text { Overdiuresis causing dehydration } \\
\pm \text { renal failure } \pm \text { electrolyte imbalance }\end{array}$ & Failure to monitor fluid balance, renal function, electrolytes etc. ${ }^{*}$ & 4 \\
\hline & Hypotension \pm hyponatraemia & & 2 \\
\hline & & Subtotal & 6 \\
\hline \multirow[t]{4}{*}{ Antiepileptics } & Toxicity & Failure to monitor phenytoin levels & 3 \\
\hline & & Failure to review patient after increasing carbamazepine dose & 1 \\
\hline & Fitting & Failure to monitor phenytoin levels & 1 \\
\hline & & Subtotal & 5 \\
\hline Other & & & $27 \dagger$ \\
\hline
\end{tabular}

at reducing medication related morbidity in primary care. ${ }^{21}$ Combining evidence from this review with results from the study presented here means that we are able to suggest the types of interventions that may be most successful in reducing the incidence of preventable drug related admissions.

In terms of prescribing related problems, our study suggests that it may be worth focusing on preventing morbidity associated with NSAIDs and antiplatelet drugs. We know that patients with two or more risk factors for gastrointestinal bleeds are at high risk, ${ }^{22}$ and that various interventions can reduce the risks of these drugs. ${ }^{23-25}$ For example, co-prescription with ulcer healing drugs can reduce the risk of gastrointestinal bleed $\mathrm{s}^{24}$ and educational interventions have been shown to improve the safety of GP prescribing. ${ }^{23}{ }^{25}$ Indeed, in a large Australian study an educational intervention focusing on NSAIDs reduced by $70 \%$ the rate of hospital admissions for upper gastrointestinal ulceration, bleeding and perforation. ${ }^{25}$

In terms of monitoring, some of the best evidence for improvements in primary care relate to the monitoring of warfarin. ${ }^{26-30}$ The results of these studies may not be applicable to other drugs, but they suggest that nurse led monitoring clinics, ${ }^{28}$ computerised decision support systems, ${ }^{27}{ }^{28}$ patient education and involvement, ${ }^{26}$ and patient selfmanagement ${ }^{29}{ }^{30}$ may help to improve control through improved monitoring. In our systematic review ${ }^{21}$ we did not find many other studies that looked at medication monitoring in primary care, and we believe that there is a need for further research in this area.

In terms of patient adherence, a number of studies have shown that improved education ${ }^{31}$ and approaches that provide greater involvement of patients in decision making ${ }^{31}{ }^{33}$ improve patient adherence and may reduce drug related admissions. However, few studies have attempted to show clinical benefits. ${ }^{34}$ Our study suggests that priority should be given to patients taking the drugs listed in table 5 .

Many of the problems identified in this study arise from medicines management in primary care, but it should be recognised that drug related admissions are relatively rare from the primary care perspective. ${ }^{35}$ For example, while NSAIDs were responsible for $12.4 \%$ of potentially preventable admissions in

Table 5 Drug classes most frequently associated with preventable drug related admissions due to adherence problems.

\begin{tabular}{llll}
\hline British National Formulary class & Adverse drug event & Problem & Number of cases \\
\hline Loop diuretics & Congestive cardiac failure & Not taking medication* & 8 \\
& Ascites & Taking too much & 1 \\
& Dehydration & Subtotal & 1 \\
& & Not taking medication & 7 \\
Antiepileptics & Fitting & Not taking medication & 7 \\
Corticosteroids & Exacerbation of asthma & Not taking medication* & 5 \\
Nitrates & Chest pain & Not taking medication & 3 \\
Insulins & Hyperglycaemia & Not eating and continuing to use same dose of insulin & 2 \\
& Hypoglycaemia & Subtotal & 5 \\
Other & & & $35 \dagger$ \\
\hline
\end{tabular}

*If a drug related admission involves more than one causative drug it may be recorded more than once in the table.

†Further details on "other" drug categories are available on the QSHC website (www.qshc.com/supplemental). 


\section{Key messages}

- Drug related morbidity was responsible for $6.5 \%$ of admissions screened by a pharmacist and $69 \%$ were considered preventable.

- The drugs most commonly associated with the preventable drug related admissions were NSAIDs, antiplatelets, antiepileptics, hypoglycaemics, diuretics, inhaled corticosteroids, cardiac glycosides and beta-blockers.

- The majority of preventable drug related admissions were caused by problems with prescribing $(35 \%)$, monitoring $(26 \%)$, or adherence $(30 \%)$.

our study, a German study estimated that NSAIDs were associated with just 5.5 hospital admissions per 10000 patients treated with these drugs in primary care. ${ }^{35}$ This may have important implications for encouraging general practitioners and health service managers that drug related admissions are important enough to justify potentially significant changes in medicines management. The types of changes outlined above could mean increased costs associated with gastroprotection for patients taking NSAIDs, more frequent monitoring for patients taking certain drugs (table 4), and more time spent educating patients and involving them in decision making. Nevertheless, these changes might improve safety for patients and protect healthcare professionals from litigation.

\section{ACKNOWLEDGEMENTS}

The authors thank Chris Love for providing the original idea for this study and for identifying the patients; Judy Cantrill and Caroline Morris, Almut Winterstein and Doug Hepler for advice on study design; Simone Brackenborough, Gail Marlow and Louise Brown for identifying the patients; Sarah Rodgers and James Turton for their help with the database; Karen Neil for helping to develop the review criteria; Michael Dewey and Simon Royal for statistical advice; Barry Fuertado from medical records for finding patient records after discharge; IM\&T at University Hospital, Nottingham for providing admissions data; and general practitioners, practice staff and patients for their contributions.

\section{Authors' affiliations}

R L Howard, A J Avery, Division of Primary Care, School of Community Health Sciences, University Hospital, Nottingham NG7 2UH, UK

P D Howard, Hayward House, Nottingham City Hospital, Hucknall Road, Nottingham NG5 IPB, UK

M Partridge, Pharmacy Department, Queens Medical Centre, University Hospital, Nottingham NG7 2UH, UK

Funding: This study was part funded by Wyeth Laboratories and part funded by NHS R\&D.

AA conceived the study and was responsible for the design along with $\mathrm{RH}$ and MP. RH collected all the data and produced all the case summaries needed for the study. $\mathrm{RH}, \mathrm{AA}$ and $\mathrm{PH}$ reviewed all of the cases. $\mathrm{RH}$ processed the data, entered it on to computer and analysed it with help from AA. All of the authors were involved in the interpretation of the results. RH and AA wrote the paper with PH and MP providing critical comments.

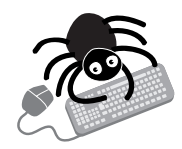

Expanded versions of tables 3, 4 and 5 are available on the QSHC website at www.qshc.com/supplemental.

\section{REFERENCES}

1 Committee on Quality of Health Care in America, Institute of Medicine. To err is human - building a safer health system. Washington, DC: National Academy Press, 1999.

2 Department of Health. An organisation with a memory. London: Department of Health, 2000.

3 Winterstein A, Sauer B, Hepler C, et al. Preventable drug-related hospital admissions. Ann Pharmacother 2002;36:1238-48.
4 Beijer HJM, de Blaey CJ. Hospitalisations caused by adverse drug reaction (ADR): a meta-analysis of observational studies. Pharm World Sci 2002;24:46-54.

5 World Health Organisation. International drug monitoring: the role of national centres. WHO Technical Report Series. 498 (9). Geneva: World Health Organisation, 1972

6 Larmour I, Dolphin RG, Baxter $\mathrm{H}$, et al. A prospective study of hospital admissions due to drug reactions. Aust J Hosp Pharm 1991;21:90-5.

7 Schneitman-Mclntire, Farnen TA, Gordon N, et al. Medication misadventures resulting in emergency department visits at an $\mathrm{HMO}$ medical center. Am J Health Syst Pharm 1996;53:1416-22.

8 Cunningham G, Dodd TRP, Grant DJ, et al. Drug-related problems in elderly patients admitted to Tayside hospitals, methods for prevention and subsequent reassessment. Age Ageing 1997;26:375-82.

9 Easton KL, Parsons BJ, Starr M, et al. The incidence of drug-related problems as a cause of hospital admissions in children. Med J Aust 1998;169:356-9.

10 Frisk PA, Cooper JW, Campbell NA. Community-hospital pharmacist detection of drug-related problems upon patient admission to small hospitals. Am J Hosp Pharm 1977;34:738-42.

11 Hallas J, Harvald B, Gram LF, et al. Drug related hospital admissions: the role of definitions and intensity of data collection, and the possibility of prevention. J Intern Med 1990;228:83-90.

12 Hepler CD, Strand LM. Opportunities and responsibilities in pharmaceutical care. Am J Hosp Pharm 1990;47:533-43.

13 World Health Organisation. International statistical classification of diseases and related health problems. 10th revision. Geneva: World Health Organisation, 1996.

14 Joint Formulary Committee. British National Formulary. 41 st edition. London: British Medical Association and Royal Pharmaceutical Society of Great Britain, 2001

15 Landis JR, Koch GG. The measurement of observer agreement for categorical data. Biometrics 1977;33:159-74.

16 Hallas J, Gram LF, Grodum E, et al. Drug related admissions to medical wards: a population based survey. Br J Clin Pharmacol 1992;33:61-8.

17 Dartnell J, Anderson R, Chohan V, et al. Hospitalisation for adverse events related to drug therapy: incidence, avoidability and costs. Med J Aust 1996; 164:659-62.

18 Hayward RA, Hofer TH. Estimating hospital deaths due to medical errors: preventability is in the eye of the reviewer. JAMA 2001;286:415-20.

19 Reason J. Human error: models and management. BM 2000;320:768-70.

20 Woods PK. The prevention of intrathecal medication errors: a report to the Chief Medical Officer. London: Department of Health, 2001.

21 Smeaton L, Sheikh A, Avery A, et al. Interventions for reducing preventable drug-related hospital admissions or preventable drug-related morbidity in primary care (protocol for a Cochrane Review). In: Cochrane Library. Issue 1. Oxford: Update Software, 2003

22 Seager JM, Hawkey CJ. ABC of the upper gastrointestinal tract. Indigestion and non-steroidal antiinflammatory drugs. $B M$ 2001;323:1236-9

23 Avorn J, Soumerai S. Improving drug therapy decisions through educational outreach. A randomised controlled trial of academically based "detailing". N Engl J Med 1983;308: 1457-63.

24 Yeomans ND, Tulassay Z, Juhasz L, et al. A comparison of omeprazole with ranitidine for ulcers associated with nonsteroidal antiinflammatory drugs. N Engl J Med 1998;338:719-26.

25 May FW, Rowett DS, Gilbert AL, et al. Outcomes of an educational-outreach service for community medical practitioners: non-steroidal anti-inflammatory drugs. Med J Aust 1999;170:471-4

26 Cromheecke $M$. Oral anticoagulation self management and management by a specialist anticoagulant clinic: a randomized cross-over comparison. Lancet 2000;356:97-102.

27 Fihn SD, McDonell MB, Vermes D, et al. A computerised intervention to improve timing of outpatient follow-up: a multicenter randomised trial in patients treated with warfarin. J Gen Intern Med 1994;9:131-9.

28 Fitzmaurice DA, Hobbs FD, Murray ET, et al. Oral anticoagulation management in primary care with the use of computerized decision support and near-patient testing: a randomized, controlled trial. Arch Intern Med 2000;160:2343-8.

29 Sawicki P. A structured teaching and self-management program for patients receiving oral anticoagulation: a randomized controlled trial. Working Group for the Study of Patient Self-Management of Oral Anti-coagulation. JAMA 1999;281:145-50.

30 Sidhu P, O'Kane H. Self-managed anticoagulation: results from a two-year prospective randomised trial with heart valve patients. Ann Thorac Surg 2001;72:1523-7.

31 Lowe C, Raynor D, Purvis J, et al. Effects of a medicine review and educational programme for older people in general practice. Br J Clin Pharmacol 2000;50:172-5.

32 Rich RW, Baldus Gray D, Beckham V, et al. Effect of a multidisciplinary intervention on medication compliance in elderly patients with congestive heart failure. Am J Med 1996;101:270-6.

33 Dowell J, Jones A, Snadden D. Exploring medication use to seek concordance with 'non-adherent' patients: a qualitative study. Br J Gen Pract 2002; 52:24-32

34 Haynes RB, McDonald H, Garg AX, et al. Interventions for helping patients to follow prescriptions for medications (Cochrane Review). In: Cochrane Library. Issue 1. Oxford: Update Software, 2003.

35 Schneeweiss S, Gottler M, Hasford J, et al. First results from an intensified monitoring system to estimate drug related hospital admissions. Br J Clin Pharmacol 2001;52:196-200. 